Original Research Paper

\title{
Statistical Modeling of Temperature Extremes Behaviour in Ghana
}

\author{
${ }^{1}$ Twumasi-Ankrah, Sampson and ${ }^{2}$ Nyantakyi, Agyei Kwadwo \\ ${ }^{1}$ Department of Mathematics, Kwame Nkrumah University of Science and Technology, Kumasi, Ghana \\ ${ }^{2}$ Ghana Institute of Management and Public Administration, Ghana
}

Article history

Received: 07-11-2018

Revised: 26-12-2018

Accepted: 7-01-2019

Corresponding Author: Twumasi-Ankrah, Sampson Department of Mathematics, Kwame Nkrumah University of Science and Technology,

Kumasi, Ghana

Tel: 233244974531

Email: sampson.ankrah@yahoo.com

\begin{abstract}
This paper focuses on modeling the extreme maximum temperature in Ghana using the extreme value theory. This is to inform decision-makers to help them plan appropriate risk mitigating measures to reduce the damage caused by drought. The block maxima with Generalized Extreme Value (GEV) and the General Pareto (GP) (with "all excesses" and decluster peaks) were used on 113 years of monthly temperature data in Ghana. Two statistical tests for stationarity, namely Augmented Dickey-Fuller (ADF) and Mann-Kendall tests were performed. In the GEV modeling, the model selection criteria (Akaike information criterion and likelihood-ratio test) and the diagnostic checking indicate that the model with linear trend in location parameter is appropriate. In fitting the GP distribution, the results from the parameter estimation show that GP with "all excesses" better fits the data than the decluster peaks. The diagnostic checking also lead to the same conclusion. The GEV estimates of the return level show that the return temperature which exceeds the maximum temperature of the observation period (36.3) starts to appear in the return period of $\mathrm{T}=20$ over years. This suggests that in 20 years to come, maximum temperature in Ghana will exceed 36.3, which may indicate a drought period.
\end{abstract}

Keywords: Extreme Value Theory, Generalized Pareto Distribution, Generalized Extreme Value Distribution, Stationarity, Return Level

\section{Introduction}

\section{Background of the Study and Problem Statement}

Variations in climatic conditions in Ghana are projected to affect the country's vital water resources, energy supplies, crop production and food security (Anon, 2015). In Ghana, the most vulnerable regions are the three northern regions, which are already experiencing increased extreme weather conditions with prolonged periods of droughts (Cameron, 2011).

Historical data have shown that temperature has been increasing over the past decades. According to McSweeney et al. (2006), mean annual temperature has increased (reports range from $+0.4^{\circ} \mathrm{C}$ over 100 years), with the strongest increase between April and July $\left(+0.27^{\circ} \mathrm{C}\right.$ per decade).

Temperature extremes, which have been known to be caused by an increase in the concentration of greenhouse gases, are natural phenomena that affect our socioeconomic activities. Extremely high temperatures negatively affect agricultural production, increase energy and water consumption (Kunkel et al., 1999). Ghana's vulnerability is largely due to the fact that crop productions are highly sensitive to climate change (Anon, 2015).

Therefore, the significance of this study rests on the fact that the agricultural and energy generation sectors in Ghana heavily depend on climatic conditions. Thus, building future situations will provide important input for farmers, researchers and local governments, who can use this information to propose adaptation measures to increase resilience. In other words, appropriate policies and plans can be drawn to prepare the general public for changes due to extreme temperatures. However, literature on extreme temperature behavior in Ghana were not much available. It was realized that not much recent information was published on the behavior of extreme temperatures in Ghana. Some of the recent studies reviewed and adapted in this work are discussed.

Hasan and Yeong (2010) modelled extreme temperature using generalized extreme value (GEV) distribution of a particular state in Malaysia. It was observed that weekly, biweekly and monthly maximums were appropriate to be fitted to the GEV model. Both 
Augmented Dickey Fuller (ADF) and Kwiatkowski, Phillips, Schmid and Shin (KPSS) stationarity tests detected no stochastic trends for maximum temperatures. However, the Mann-Kendall (MK) test showed that all three selection periods had a decreasing trend. When the KolmogorovSmirnov and Anderson-Darling goodness of fit tests were conducted, they revealed that all three-selection period maximum values converged to the GEV distribution.

Hasan et al. (2013) extended her work to fit the monthly maximum temperature to the GEV distribution for a number of stations in Malaysia. They tested for trend using the Mann-Kendall test and it showed the existence of trend for some stations and absence in other stations. Therefore, the annual maximum temperatures were modelled by applying both the stationary and nonstationary GEV distribution to the different stations. They concluded that the non-stationary model was the best because it explained much of the variation in the data.

Also, Ayuketang and Joseph (2015) modelled extreme temperature in Cameroon by using the same GEV distribution. They used five different selection periods namely monthly, bi-monthly, quarterly, halfyearly and yearlyin order to have enough data for modeling purposes. When these selection periods were fitted to the GEV distribution, they found that monthly, bimonthly and quarterly selection periods were the best. The Mann-Kendall trend test showed that all the selection periods were decreasing as time increases. In addition, the Kolmogorov-Smirnov and AndersonDarling goodness of fit tests revealed that all selection periods converged to the GEV distribution with monthly maximum having the best convergence.

Bommier (2014) used extreme value theory based on the block maxima and Peak Over Threshold (POT) approaches to analyze monthly extreme temperatures of Uppsala in Sweden. In comparing the results of the two estimation methods on the same time series data different values were observed for the return periods. For maxima, estimates for the return periods were larger with the GEV model compare to the POT method, whereas for the minima, the GEV model gave lower return period estimates than the POT method.

In Ghana, among the most recent related studies reviewed in this study is Mori (2016) who used both the Generalized Extreme Value (GEV) and Generalized Pareto Distributions (GPD) to model monthly extreme temperature behavior of the Upper East Region. The results revealed that the GEV model was better in modelling extreme temperature behavior because it has the least AIC and BIC values of -1003.6050 and 991.7600 , respectively. Also, they concluded that the maximum temperature returns showed an increasing trend for longer return periods.

Another study on Ghana is by Nkrumah (2017) which indicated that the extreme occurrence of temperature can be modelled using Weibull and Frechet family of distributions for the Accra, Ashanti and Northern regions. A maximum temperature of $34.7^{\circ} \mathrm{C}, 34.66^{\circ} \mathrm{C}$ and $39.6^{\circ} \mathrm{C}$ was predicted to occur in Accra, Ashanti and Northern regions, respectively once every five years.

The fundamental issue in these two studies on Ghana is that for the GEV approach, though there was the presence of trend, it was not captured in the modelling process. Also, these studies were concentrated on four regions in Ghana. Thus, a study that incorporates the non-stationary nature of the data and also looks at the whole country's extreme temperature behavior will be helpful. Hence, the study seeks to apply both GEV and GPD to maximum temperature in Ghana by considering the non-stationary nature too. This will specifically help us to estimate the likelihood of the increment of these temperature values in the future. The GEV and GDP distributions are the two commonly used distributions for modeling extremal events (Coles, 2001).

\section{Methods and Materials}

\section{Data Source}

The monthly maximum temperature for the whole Ghana for the period 1900-2013 was obtained from the Climatic Research Unit (University of East Anglia) which is developed in conjunction with the Hadley Centre (UK Met Office).

In this study, the two approaches of modeling extreme events are used, namely generalized extreme vale and generalized pareto distribution. Three blocking methods are considered, namely, monthly, annual and hot. Annual maxima are the maximum temperature value in each year over the entire study period. For the hot spell, we construct a data frame of maximum temperature in the summer period between June 15 and September 15 (92 day) every year (Sheng, 2012).

The empirical analysis is performed using the following $\mathrm{R}$ packages evd, ismev, POT, extRemes and MASS.

\section{Generalized Extreme Value (GEV)}

Consider an independent sequence $X_{1}, \ldots, X_{n}$, the block maximum is generated by $M_{n}=\max \left(X_{1}, \ldots, X_{n}\right)$, having a common distribution function $F$. The distribution of block maximum $G(\mathrm{z})$ is the Generalized Extreme Value (GEV) distribution:

$G(z)=\exp \left\{-\left[1+\xi\left(\frac{z-\mu}{\sigma}\right)\right]^{-1 / \xi}\right\}$,

There are three parameters in Equation (1):

1) Location parameter, $\mu$, which is the center of the GEV distribution.

2) Scale parameter, $\sigma$, which determines the size of deviations of $\mu$, and 
3) Shape parameter, $\zeta$ shows how rapidly the upper tail decays.

Here, positive $\zeta$ implies a heavy tail while negative one implies a bounded tail and the limit of $\zeta \rightarrow 0$ implies an exponential tail (Coles, 2001). According to Coles (2001), this information is represented by the three families of distribution function as in Equations (2)-(4):

- $\quad$ Type I, Gumbel family which corresponds to case $\zeta$ $=0$, i.e., GEV family with limit:

$$
\mathrm{G}(\mathrm{z})=\exp \left\{-\exp \left[-\left(\frac{\mathrm{z}-\mathrm{b}}{\mathrm{a}}\right)\right]\right\},-\infty<\mathrm{z}<\infty
$$

- $\quad$ Type II, Fréchet family which corresponds to case $\zeta$ $>0$ of GEV family:

$$
G(z)=\exp \begin{cases}0, & z \leq b \\ -\exp \left\{-\left(\frac{z-b}{a}\right)^{-a}\right\} & z>b\end{cases}
$$

- Type III, Weibull family which corresponds to case $\zeta<0$ of GEV family:

$$
G(z)=\exp \begin{cases}\exp \left\{-\left(\frac{z-b}{a}\right)^{a}\right\}, & z \leq b \\ 1, & z>b\end{cases}
$$

\section{Trend in GEV}

In this study, two tests of stationarity, namely, MannKendall and Augmented Dickey-Fuller are used to evaluate the existence of trend in our data. The MannKendall nonparametric test is used to detect trend in environmental applications (Ryden, 2011). Null hypothesis $\left(H_{0}\right)$ is that there exists no trend and alternative hypothesis indicates the existence of trend.

A non-stationary process means that the trend exist in the sequence $X_{1}, \ldots, X_{n}$. In this study, four models are considered under the GEV distribution (i.e.; model 1 (M1) is normal GEV model, model 2 (M2) is linear trend in location parameter, model 3 (M3) is quadratic trend in location parameter, model 4 (M4) is linear trend in scale parameter). The later three models are used to capture the trend nature in the data. These models can be represented as:

$$
\begin{aligned}
& M 2: \mu(t)=\beta_{0}+\beta_{1} t \\
& M 3: \mu(t)=\beta_{0}+\beta_{1} t+\beta_{1}^{2} t \\
& M 4: \sigma(t)=\sigma_{0}+\sigma_{1} t \\
& \xi(t)=\xi
\end{aligned}
$$

\section{Return Values}

Return values contain two quantities: return period $1 / p$ and return level (recurrence interval) $Z_{p}$. According to Sheng (2012), for annual maxima example, return level is an estimated high value of annual maxima temperature which is expected to be exceeded in any year during return period $1 / p$ with probability $p$ where $0<p<0$.

\section{Model Diagnostics}

The goodness-of-fit of the model is assessed by the following methods:

\section{P-P plot}

Probability-Probability $(P-P)$ plot compares the theoretical and empirical Cumulative Distribution Function (CDF) to assess if a fitted result is a reasonable model. An acceptable model leads the $P-P$ plot close to a diagonal line CDF.

\section{Q-Qplot}

Quantile-quantile $(Q-Q)$ plot assesses the adequacy of a fitted distribution by comparing the $1 /(n+1) t h$ quantile driving from the theoretical and empirical distribution. A reasonable model leads the $Q-Q$ plot close to a diagonal line.

\section{Likelihood Ratio Test}

The deviance function is used to test between choices of model:

$$
D(\theta)=2\left\{l\left(\hat{\theta}_{0}\right)-l(\theta)\right\}
$$

where, $l$ is the log-likelihood function, $\hat{\theta}_{0}$ is the maximum likelihood estimator of parameter $\theta$ which we want to estimate. A low P-value rejects the null hypothesis of equal parameters.

\section{Generalized Pareto Distribution (GPD)}

Let $u$ be the reasonable threshold which can be determined by some methods. The exceedances over the threshold $u$, i.e. $h=X-u$ can be modeled by the GPD, which is written as:

$$
\mathrm{H}(\mathrm{h}=\mathrm{x}-\mathrm{u})=1-\left[1+\frac{\xi \mathrm{h}}{\sigma_{\mathrm{u}}}\right]^{-1 / \xi}, \mathrm{x}>\mathrm{u}, 1+\frac{\xi \mathrm{h}}{\sigma_{\mathrm{u}}}>0
$$

where, $h$ is the exceedance, $u$ is the threshold value, $\sigma_{u}$ is the scale parameter over the threshold and $\zeta$ is the shape parameter. 


\section{Threshold Selection}

The selection of reasonable threshold is the first step in modeling the GPD. In selecting the threshold, three points are considered:

i. Empirical quantile

The simplest way to select a threshold is to choose from the raw data at a specified empirical quantile in the range of $90 \%$ to $97 \%$ (Sheng, 2012)

ii. Mean excess plot

In this plot, estimator of the shape parameter should appear approximately linear in threshold $u$ above a reasonable $u_{0}$

iii. Stability checking of shape parameters

Some values of threshold candidates can be selected based on appropriate methods, when we can then fit parameters in a GPD for each of the threshold. A suitable threshold can be chosen when the estimators of the shape parameter $\zeta$ keep stable above the threshold.

In this study two approaches are used to model the GPD:

\section{All Excess over Threshold}

In extreme value theory, all excesses over a selected threshold are extreme events. Here, all excesses are fitted using the GPD.

\section{Cluster Peaks}

Clusters are defined with a selected threshold $u$ and a choice of cluster width $r$; a cluster ends by $r$ consecutive observation values which fall below the threshold. It is necessary to decluster data in order to analyze cluster length or dependent relationship in clusters. Coles (2001) outlined the procedure of decluttering as:

- Define clusters of exceedances with a threshold and cluster width

- Identifying the maximum excess within each cluster

- Fitting the conditional excess distribution given by the GPD under the assumption of independence in cluster maxima

\section{Results and Discussion}

As mentioned, this study is based on the series of monthly maximum temperature data for the whole Ghana, which was collected during 1900-2013. This makes the total observation to be 1356 , which shows that the blocks are enough to fit GEV distribution. The analysis was done for three different blocks of selection periods, namely, monthly, annual and hot (the hottest period in a year). Two extreme events modeling approaches namely GEV and GDP are studied and compared in order to get an appropriate approach for the temperature maxima in Ghana.

\section{Descriptive Measures}

Table 1 shows the main descriptive measures for the monthly maximum temperature at the various selection periods. The original monthly maximum temperature has the largest standard deviation of 2.22 , which indicates a varied monthly maximum temperature. However, the standard deviation is quite smaller in the remaining selection period.

The skewness is slightly negative on the monthly selection period but positive on the annual and seems normal on the hot selection period of maximum temperature. This observation suggests that the maximum temperature for monthly data fit a distribution which is relatively long left tailed. However, the annual selection period suggests a distribution with a right tail which is relatively longer than the left tail.

The P-values of the two normality tests (i.e. anderson-Darling Test and Shapiro-Wilk Test) for the monthly and annual maximum temperature reject the null hypothesis of a normal distribution while the hot period fails to reject the null hypothesis. Thus, the hot selection period is not appropriate for extreme value analysis.

\section{Unit Root Test for Stationarity}

From Fig. 1, the data looks stationary over the monthly selection period but there seem to be a strong evident of trends in the annual selection period. To verify the stationarity, we used the Augmented DickeyFuller Test.

Table 2, the P-value (0.23) associated with the annual selection period is greater than 0.05 significance level and therefore we can conclude that the annual period data is not stationary. However, the P-value (0.02) associated with the monthly period is less than the 0.05 significance level and therefore it is stationary. We assume that the pattern of variation in the annual selection period has been changing over the observation period, but the monthly period has been constant over the observed period.

Table 1: Basic descriptive statistics of maximum temperature

\begin{tabular}{lllllllll}
\hline Period & N & Min. & Max. & Mean & S. Dev & Skewness & Anderson-Darling Test & Shapiro-Wilk Test \\
\hline Monthly & 1356 & 27.7 & 37.4 & 32.43 & 2.22 & -0.12 & $15.88(0.00)$ & $0.96(0.00)$ \\
Annual & 113 & 34.1 & 37.4 & 35.66 & 0.66 & 0.49 & $1.18(0.00)$ & $0.97(0.01)$ \\
Hot & 113 & 32.9 & 37.4 & 35.4 & 0.74 & 0.00 & $0.68(0.07)$ & $0.98(0.15)$ \\
\hline
\end{tabular}


Table 2: Unit Root (ADF) test for maximum temperature

\begin{tabular}{llll}
\hline Period & Test statistics & P-value & Decision \\
\hline Monthly & -3.47 & 0.02 & Reject Null hypothesis. Series is stationary \\
Annual & -2.82 & 0.23 & Do not reject Null hypothesis. Series is not stationary \\
\hline
\end{tabular}
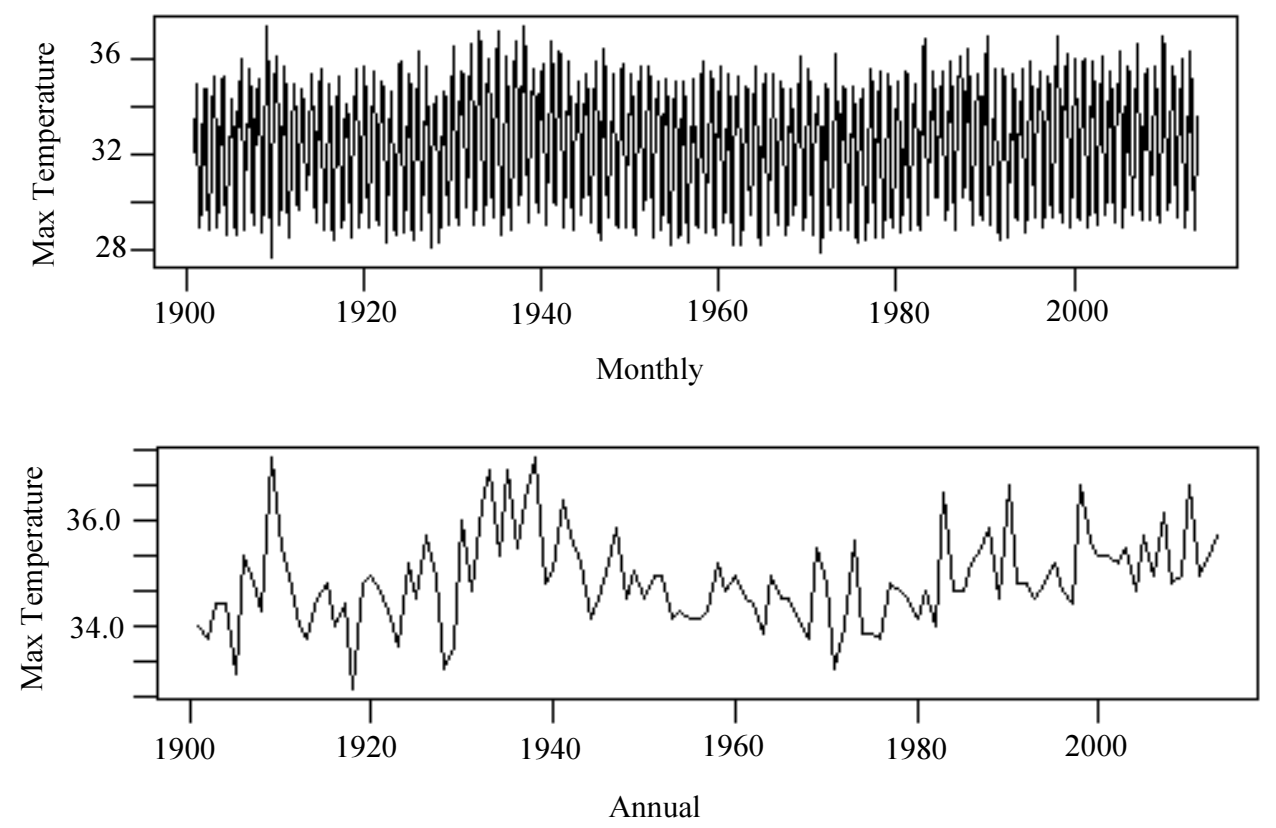

Fig. 1: Time series plot of maximum temperature in Ghana over the period 1900-2013 for the monthly and annual periods

The Mann-Kendall (MK) test was performed under the null hypothesis of absence of trends to verify the results and the results are as in Table 3 .

The annual maximum temperature shows the existence of a trends time increases, while the monthly has no trend. This result suggest that we ought to model for both stationarity and non-stationarity in this study.

\section{GEV Model Fitting}

Here, we will consider parameter estimation for only the annual selection period, since the monthly period is the original series and the hot selection period is normally distributed. For the annual selection period, four models (M1, M2, M3 and M4) are considered.

Fitting the GEV distribution to the annual maximums leads to the maximum likelihood estimates as shown in Table 4.

\section{Model Selection}

For the annual selection periods, two model selection criteria namely Akaike Information Criterion (AIC) and likelihood-ratio test are used to select the adequate model. The model selection results for the annual maximum temperature are presented in Table 5.
The AIC considered model 2 as the best or adequate model. Furthermore, the likelihood-ratio test indicates that model 2 is better than model 1, 3 and 4. Thus, we conclude that model 2 , where $\mu$ vary linearly with respect to time and other parameters are constants, is the best model.

\section{Model Diagnostics}

Figure 2 shows the model diagnostics for annual maximums for Model 2.The residual probability and quantile plots are displayed for Model 2 in Fig. 2. These plots suggest that Model 2 (for annual maximums) has a good fit.

\section{Return Level Estimate}

The highest monthly temperature for the 15-year observation period is 36.3. Return levels are used to predict the probability that a monthly maximum temperature exceeding 36.3 will occur in a longer period.

The return levels for annual selection periods with their $95 \%$ confidence intervals which are obtained by profile likelihood are displayed in brackets in Table 6. It can be observed that return level estimates increase as the return periods increase. 
Twumasi-Ankrah, Sampson and Nyantakyi, Agyei Kwadwo / Journal of Mathematics and Statistics 2018, Volume 14: 275.284 DOI: $10.3844 / j m s s p .2018 .275 .284$

Table 3: Mann-Kendall test of trend

\begin{tabular}{llll}
\hline Period & Test statistics & (2-sided) P-value & Decision \\
\hline Monthly & 0.02 & 0.27 & Do not reject Null hypothesis. No trend in the series \\
Annual & 0.16 & 0.02 & Reject Null hypothesis, there is trend in the series \\
\hline
\end{tabular}

Table 4: Parameter Estimates for Annual Maximum Temperature

\begin{tabular}{lllll}
\hline Parameters & Model 1 & Model 2 & Model 3 & Model 4 \\
\hline$\mu$ & $35.39(0.06)$ & & & $35.49(0.062)$ \\
$\sigma$ & $0.60(0.04)$ & $0.56(0.04)$ & $0.55(0.035)$ & $-0.138(0.079)$ \\
$\zeta$ & $-0.15(0.057)$ & $-0.093(0.061)$ & $-0.0833(0.0093)$ & $0.72(0.09)$ \\
$\beta_{0}$ & & $35.07(0.12)$ & $35.18(0.11)$ & $-0.0024(0.001)$ \\
$\beta_{1}$ & & $0.0054(0.0017)$ & $-0.0012(0.0016)$ & -110.77 \\
$\beta_{1}^{2}$ & & & $0.0000593(\mathrm{NaN})$ & -106.03 \\
LLV & -110.84 & -106.61 & & \\
\hline
\end{tabular}

Table 5: Model Selection Criteria for Annual Maximum Temperature

\begin{tabular}{lll}
\hline Criteria & Model & Value \\
\hline AIC & M1 & 227.68 \\
& M2 & $221.21^{*}$ \\
& M3 & 222.05 \\
Likelihood Ratio Test & M4 & 226.42 \\
& & Test statistic \& P-value \\
& M1 vrs M2 & $8.46(0.00)$ \\
M1 vrs M3 & $9.63(0.00)$ \\
M1 vrs M4 & $3.26(0.07)$ \\
M2 vrs M3 & $1.16(0.28)$ \\
M2 vrs M4 & $-5.21(0.99)$ \\
\hline
\end{tabular}

Table 6: Return Level Estimate of Annual Maximum for $\mathrm{T}=2,20,100$ with $95 \%$ confidence interval

\begin{tabular}{lllc}
\hline Period & $\mathrm{T}=2$ & $\mathrm{~T}=20$ & $\mathrm{~T}=100$ \\
\hline Annual & 35.38 & 36.84 & 37.45 \\
& $(35.28,35.89)$ & $(36.53,37.14)$ & $(37.17,37.79)$ \\
\hline
\end{tabular}
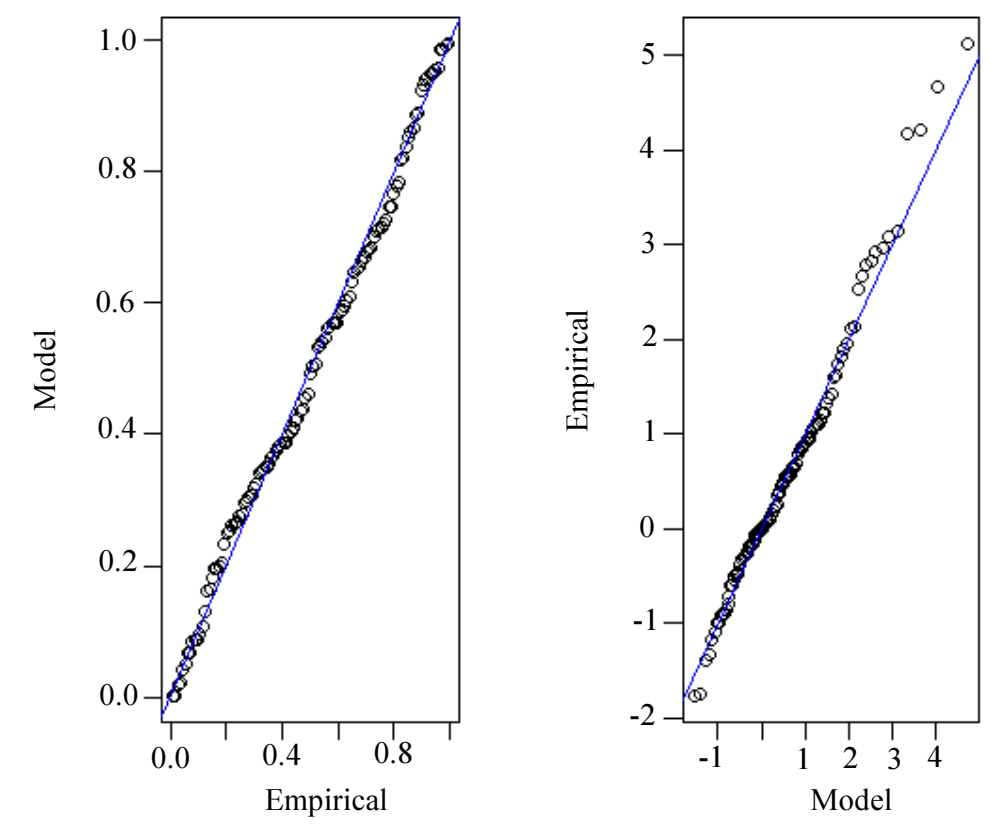

Fig. 2: Model diagnostic for annual maximum 
It is observed that the temperature which exceeds the maximum temperature (36.3) of the observation period appears in the confidence interval of $\mathrm{T}=20,100$ for the selection period (annual). This suggests that in 20 years to come, maximum temperature in Ghana will exceed 36.3.

\section{Modeling using GPD}

In extreme event modeling using GEV, the data set is built on block maxima and the maximum value in each block is used to estimate the parameters. However, in the modeling of estimation of GPD and POT approach, the data set is based on all of the raw data where a threshold value is identified and all data values above the threshold is considered as the maxima and used in the modeling.

\section{Threshold Selection}

The selection of an appropriate threshold forms the basis of the POT method. Three methods are used to select an appropriate threshold for the monthly temperature data. We will start with Mean Excess plot for Maxima.
Figure 3 shows the mean residual life plot with approximate $95 \%$ confidence intervals for the monthly temperature data. By taking the confidence intervals into account, the graph appears to decay sharply from $u=32$ to $u=35.3$, beyond which it is approximately linear until $u=36$. Hence, it is probably better to conclude that there is some evidence for linearity above $u=35.3$ and to work initially with a threshold set at $u=35.3$.

For the Threshold Candidates, we fit candidate GPD with varying threshold which is based on percentile. The choice of appropriate threshold will be the first of these candidate values for which there is stability in the GPD parameter estimates from that point on. If no such stability is seen, then the fitting of a GPD to the tail may not be reasonable. From Table 7, we begin to see stability in the parameter estimates with the threshold set to the 10th percentile of the data set and this is our choice for the threshold. Therefore, an appropriate threshold is $u=35.3$.

Table 7: Threshold Candidates: with associated GPD parameter estimates

\begin{tabular}{lllll}
\hline & $\begin{array}{l}\text { Threshold } \\
\text { Candidate }\end{array}$ & $\begin{array}{l}\text { No. of value } \\
\text { above threshold }\end{array}$ & GPD Parameter estimates \\
\hline Percentile & 35.3 & 135 & Shape & Scale \\
\hline 10th & 35.4 & 123 & -0.21 & 0.69 \\
9th & 35.5 & 108 & -0.21 & 0.69 \\
6th & 35.6 & 81 & -0.25 & 0.69 \\
5th & 35.7 & 67 & -0.32 & 0.75 \\
4th & 35.8 & 54 & -0.37 & 0.78 \\
3rd & 36.0 & 40 & -0.59 & 0.47 \\
\hline
\end{tabular}

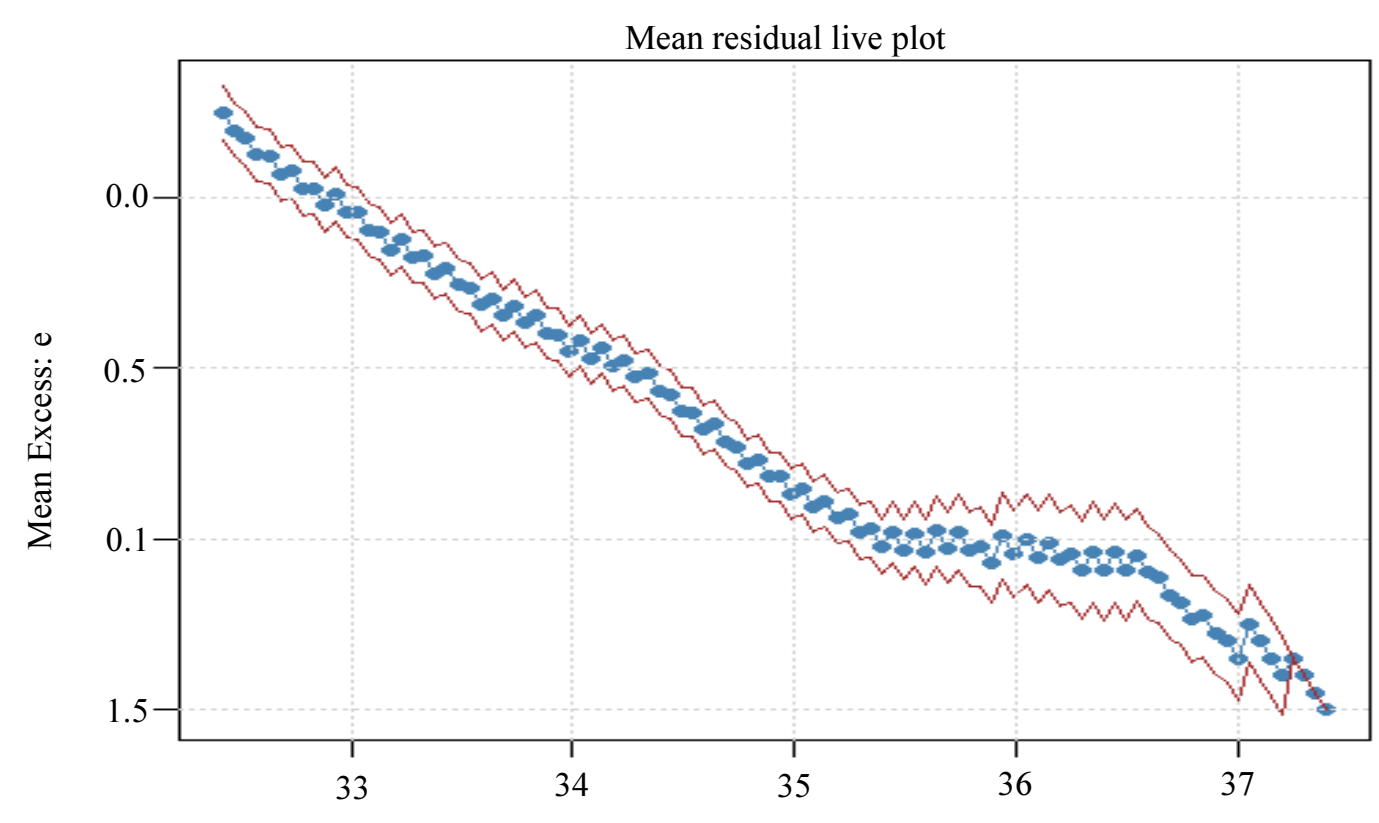

Threshold: u

Fig. 3: Mean residual life plot for maxima 
The Threshold stability plot which compares the shape and scale parameters for a series of threshold values are in Fig. 4, it can be seen that the threshold with the minimum scale parameter and highest shape parameter is $u=35.3$. Hence, $u=35.3$ can be chosen as the threshold.

Hence, the selected threshold of $u=35.3$ appears reasonable for the data.

\section{Model Estimates for all Excesses and Cluster Peaks}

The maximum likelihood estimates of the GPD scale and shape parameters $\hat{\sigma}$ and $\xi$, along with the associated $95 \%$ confidence intervals, fitted to the set of all excesses (threshold) and cluster peak excesses are given in Table 8. The maximum likelihood estimate for both all excesses and cluster peaks corresponds, to a bounded distribution (since $\hat{\xi}<0$ ) and the evidence for this is reasonably strong, since the $95 \%$ interval for $\hat{\xi}$ is in the negative domain.

The point estimates for the cluster peaks scale parameter is not significantly different from zero. However, the shape parameters are not significantly different in both cases. This makes the GPD fitted to the all excesses approach more appropriate than the cluster peak approach.
The rate of excess in the GPD fitted is higher in the all excesses approach; that is the rate at which the series exceeds the threshold of 35.3 is higher in the all excesses approach. Note that the AIC values for these two fitted models are not comparable since they are fitted to different sets of data.

\section{Diagnostic Checking}

Figure $5 \mathrm{a}$ and $5 \mathrm{~b}$, the diagnostic plots for the fitted generalized Pareto model with threshold $u=35.3$ and cluster peaks are shown. It is obvious that, none of the plots gives any real cause for concern about the quality of the fitted model.

\section{Return level Estimates for all Excesses and Cluster Peaks}

Comparing return levels estimated by GPD for all excesses data and cluster peaks data given in Table 9 and Fig. 6, there are no significant differences at long periods (after $\mathrm{T}=30$ ). The obvious differences occur at the short return periods (before $\mathrm{T}=50$ ).

Note that the return levels computed from the declustered data refer to the occurrence of cluster maxima, rather than all threshold excesses and need to be interpreted accordingly.

Table 8: Maximum likelihood estimates and associated 95\% confidence intervals, for the GPD scale and shape parameters

\begin{tabular}{llllll}
\hline & $\hat{\sigma}$ & $\hat{\xi}$ & LLV & AIC & Rate of excess \\
\hline Cluster Peaks 95\% C.I & $-0.15(0.15)[-0.44,0.14]$ & $-0.30(0.11)[-0.52,-0.08]$ & -43.4 & 90.8 & 0.059 \\
All Excesses 95\% C.I & $0.69(0.08)[0.53,0.85]$ & $-0.21(0.08)[-0.37,-0.05]$ & -56.08 & 116.7 & 0.098 \\
\hline
\end{tabular}

Table 9: Return level estimate of monthly temperature

\begin{tabular}{lllllll}
\hline Return Level & $\mathrm{T}=20$ & $\mathrm{~T}=30$ & $\mathrm{~T}=50$ & $\mathrm{~T}=100$ & $\mathrm{~T}=200$ & $\mathrm{~T}=1000$ \\
\hline Cluster Peaks & 35.4 & 35.7 & 36.1 & 36.5 & 36.8 & 37.3 \\
All Excesses & 35.7 & 36 & 36.2 & 36.6 & 36.8 & 37.3 \\
\hline
\end{tabular}

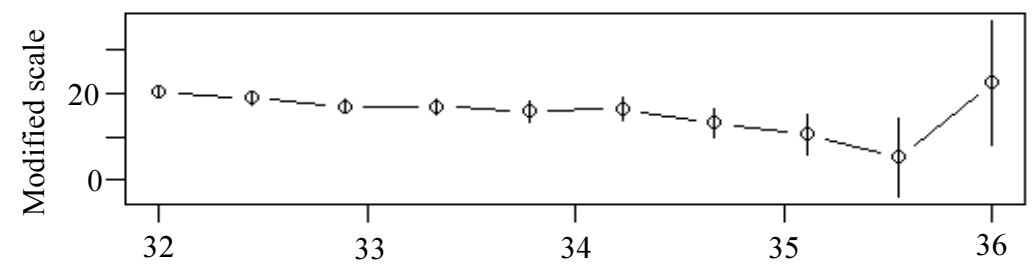

Threshold

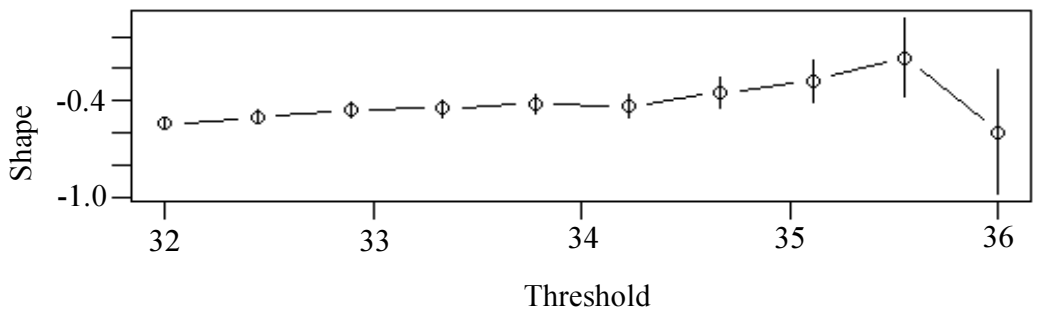

Fig. 4: Parameter estimates against threshold for monthly temperature 

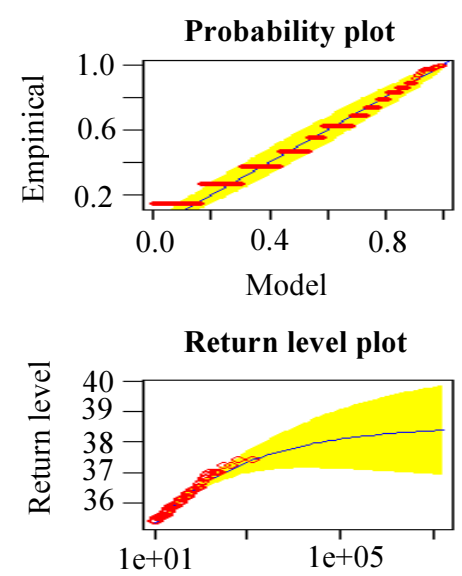

Return period
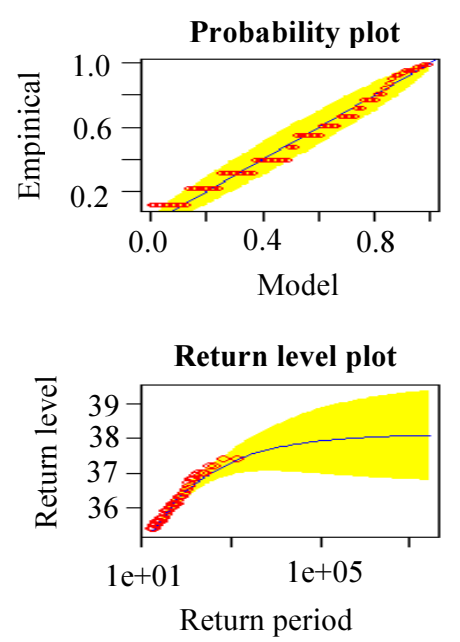
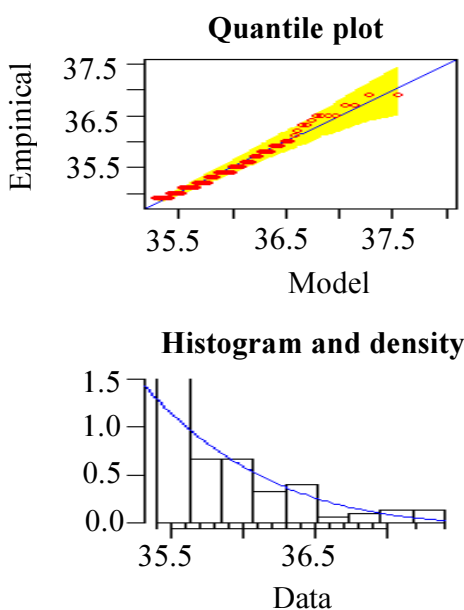

(a)

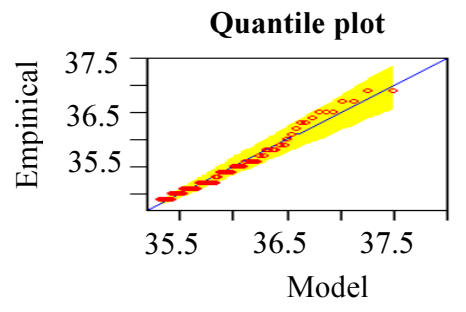

Histogram and density

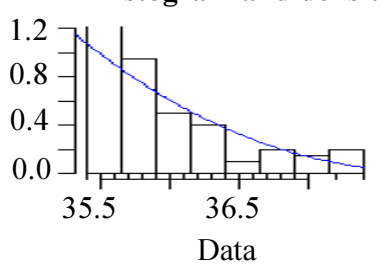

(b)

Fig. 5: (a) Diagnostic plots for threshold excess model fitted to monthly temperature data (b) Diagnostic plots for cluster peaks model fitted to monthly temperature data

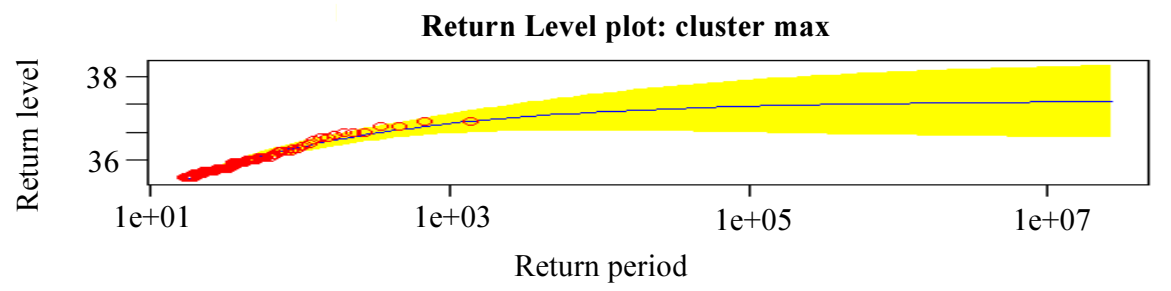

Return Level plot: Original series

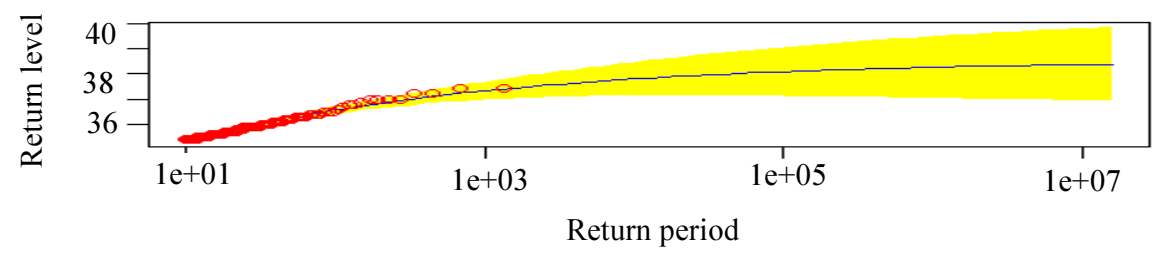

Fig. 6: Return level plots original series (TOP) and cluster maximum (BOTTOM) 
The results are also consistent with Hasan and Yeong (2010) who conducted a study using maximum temperature in Malaysia. Their study also revealed that the GEV model was more appropriate in modelling temperature data rather than the GPD model.

\section{Conclusion}

The purpose of this study is to provide statistical knowledge about the behavior of extreme temperatures for policy-makers so that appropriate risk mitigating measures to reduce the damage caused by drought. The Block Maxima model with GEV and the GP (with all excesses and decluster peaks) were used on 113 years of monthly temperature data in Ghana.

In the GEV modeling, the model selection criteria (AIC and likelihood-ratio test) and the diagnostic checking indicate that the model with linear trend in location parameter is appropriate. Also, in fitting the GP distribution, the results from the parameter estimation shows that GP with all excesses better fits the data than the decluster peaks.

Overall, by considering the diagnostic checking (of both best models under GEV and GP), the GEV model with linear trend in location parameter seems to fit the data better than the all excesses GP model.

The GEV estimates of the return level show that the return temperature which exceeds the maximum temperature of the observation period (36.3) starts to appear in the return period of $\mathrm{T}=20$. This suggests that in 20 years to come, maximum temperature in Ghana may exceed 36.3; indicating a drought period.

This paper suggests the use of GEV approach in modeling monthly temperature in Ghana. The limitation of this study is that other estimation techniques apart from MLE should be considered for this purpose.

\section{Acknowledgement}

The author would like to thank the reviewers for their helpful comments and suggestions.

\section{Author's Contributions}

Twumasi-Ankrah, Sampson: Designed the study and coordinated the data analysis, interpretation and write-up

Nyantakyi, Agyei Kwadwo: Acquisition of data and literature review.

All authors contributed to the development and publication of this work. All authors read and approved the final manuscript for publication.

\section{Ethics}

The author declares that there is no conflict of interest regarding the publication of this manuscript.

\section{References}

Anon, 2015. Climate change profile Ghana. Netherlands Commission for Environmental Assessment.

Ayuketang, N. and E. Joseph, 2015. Modelling extreme temperature in cameroon using generalized extreme value distribution. Int. J. Physical Mathematical Sci.

Bommier, E., 2014. Peaks-over-threshold modelling of environmental data. University of Uppsala, Sweden. U. U. D. M. Project Report 2014.33.

Cameron, C., 2011. Climate change finance and aid effectiveness: Ghana case study. OECD.

Coles, S., 2001. An introduction to Statistical Modeling of Extreme Values, 1st Edn. Springer.

Hasan, H. and W.C. Yeong, 2010. Extreme value modelling and prediction of extreme rainfall: A case study of Penang. AIP Conf. Proc., V1309: 372-393.

Hasan, H., N. Salam and B.M. Adam, 2013. modelling extreme temperature using generalized extreme value distribution. Int. J. Mathem. Computational Sci., 7: 983-988.

Kunkel, K.E., R.A. Pielke Jr. and S.A. Changnon, 1999. Temporal fluctuations in weather and climate extremes that cause economic and human health impacts: A review. Bulletin Am. Meteorological Society, 80: 1077-1098.

McSweeney, C., M. New and G. Lizcano, 2006. UNDP Climate change country profiles. Ghana.

Mori, K.W., 2016. Modelling extreme temperature behaviour in upper east region, Ghana. University for Development Studies. An Unpublished M.Sc. Thesis.

Nkrumah, S., 2017. Extreme value analysis of temperature and rainfall: Case study of some selected regions in Ghana. University of Ghana. An Unpublished M.Sc. Thesis.

Ryden, J., 2011. Statistical analysis of temperature extremes in long-time series from Uppsala. J. Theoretical Applied Climatology, 105: 193-197.

Sheng, G., 2012. Estimation of hot and cold spells with extreme value theory. U. U. D. M. Project Report 2012:19. 